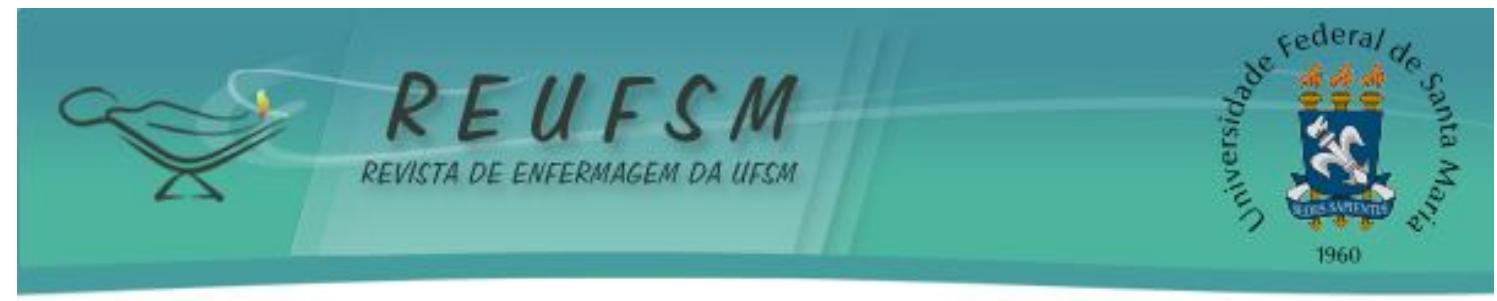

ARTIGO ORIGINAL

\title{
CARACTERIZAÇÃO DE CRIANÇAS EM TRATAMENTO CIRÚRGICO EM UM HOSPITAL ESCOLA NO SUL DO BRASIL
}

\section{CHARACTERIZATION OF CHILDREN IN SURGICAL TREAT MENT IN A TEACHER HOSPIT AL IN SOUT HERN BRAZIL}

\section{CARACTERÍST ICAS DE LOS NIÑOS EN TRATAMIENTO QUIRÚRGICO EN UN HOSPIT AL ESCUELA DEL SUR DE BRASIL}

\author{
Andressa da Silveira ${ }^{1}$ \\ Eliane Tatsch Neves ${ }^{2}$ \\ Anaiara Fortes Famoso ${ }^{3}$ \\ J oanita Cechin Donaduzzi ${ }^{4}$ \\ Carolina Frescura J unges ${ }^{5}$ \\ Kellen Cervo Zamberlan ${ }^{6}$
}

RESUMO: Trata-se de um estudo descritivo, quantitativo e retrospectivo que teve por obj etivo caracterizar crianças submetidas a tratamento cirúrgico em um hospital de ensino no sul do Brasil. Por meio da análise documental foram analisados os prontuários das crianças que internaram no Hospital Universitário de Santa Maria (HUSM) no período de janeiro a dezembro de 2006, totalizando 107 prontuários. Os dados foram submetidos a análise estatística simples. Constatou-se que a maioria das crianças internadas no período eram do sexo masculino, estavam em idade escolar e eram procedentes da região central do Rio Grande do Sul, sendo o diagnóstico mais comum as apendicites agudas. A média de dias de internação ficou em torno de cinco dias. 88\% das crianças tiveram a mãe como principal cuidadora. Por fim, recomenda-se atenção da equipe de enfermagem em relação à quantidade e qualidade dos registros nos prontuários.

Descritores: Enfermagem pediátrica; Cuidados de enfermagem; Saúde da criança; Procedimentos cirúrgicos operatórios.

ABSTRACT: This is a quantitative, descriptive and retrospective study that aimed to characterize children undergoing surgery in a teaching hospital in southern Brazil. Using the documentary analysis the medical records of children who were hospitalized at University Hospital of Santa Maria (HUSM) in the period J anuary to December 2006 were analyzed, totaling 107 charts. Data were submitted to simple statistical analysis. It was found that the majority of children hospitalized in the period was male, in school age and from the central region of Rio Grande do Sul. The most common diagnosis was acute appendicitis. The average hospital stay was about five days. $88 \%$ of children had their

\footnotetext{
${ }^{1}$ Enfermeira. Mestranda do Programa de Pós Graduação em Enfermagem da Universidade Federal de Santa Maria (UFSM). Membro do Grupo de Pesquisa Cuidado a Pessoas, Famílias e Soci edade (PEFAS). Bolsista REUNI. E-mail: andressadasilveira@gmail.com

2 Enfermeira Pediatra. Doutora em Enfermagem, Professora Adj unta do Curso de Enfermagem e do Programa de Pós Graduação em Enfermagem da Universidade Federal de Santa Maria (UFSM). Membro do Grupo de Pesquisa Cuidado a Pessoas, Famílias e Sociedade (PEFAS). E-mail: elianeves03@gmail.com

3 Enfermeira. Membro do Grupo de Pesquisa Cuidado a Pessoas, Famílias e Sociedade (PEFAS). E-mail: affamoso@gmail.com

${ }^{4}$ Enfermeira. Mestre em Enfermagem pela Universidade Federal de Santa Maria (UFSM). Membro do Grupo de Pesquisa Cuidado a Pessoas, Famílias e Sociedade (PEFAS). E-mail: joanitanurse@yahoo.com. br

5 Enfermeira. Mestre em Enfermagem pela Universidade Federal de Santa Maria (UFSM). E-mail: cfj unges@hotmail.com

${ }^{6}$ Acadêmica de Enfermagem do $8^{\circ}$ semestre da Universidade Federal de Santa Maria (UFSM). Membro do Grupo de Pesquisa Cuidado a Pessoas, Famílias e Sociedade (PEFAS). E-mail: kellencz@hotmail.com
} 


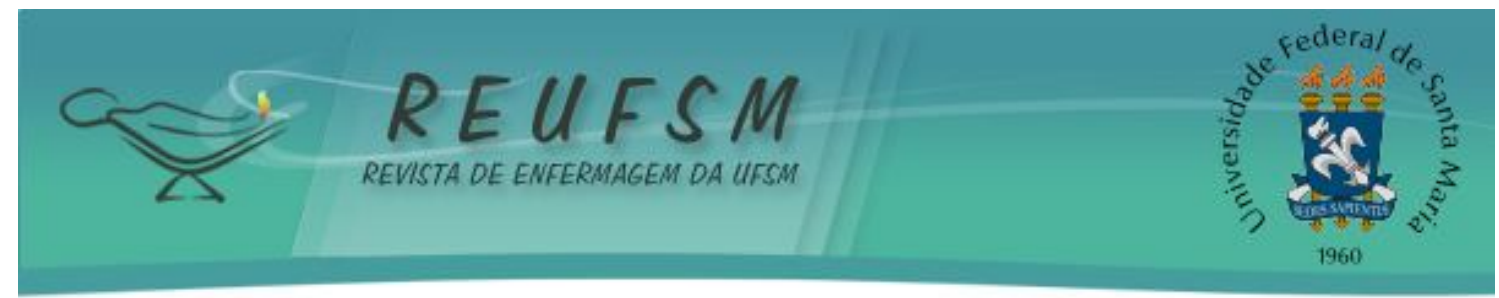

mother as primary caregiver. Finally, it is recommended to the attention of the nursing staff in relation to quantity and quality of records in order.

Descriptors: Pediatric nursing; Nursing care; Child's health; Surgical procedures operative.

RESUMEN: Este es un estudio descriptivo, cuantitativo y retrospectivo que tuvo como objetivo caracterizar a los niños sometidos a cirugía en un hospital universitario en el sur de Brasil. A través del análisis documental, se analizaron los expedientes médicos de los niños que fueron hospitalizados en el Hospital Universitario de Santa Maria (HUSM) en el período de enero a diciembre de 2006, un total de 107. Los datos fueron sometidos a un análisis estadístico sencillo. Se encontró para la mayoría de los niños hospitalizados en el período, varones, en edad escolar y provenientes de la región central de Rio Grande do Sul, el diagnóstico de apendicitis aguda. La estancia media hospitalaria fue de unos cinco días. El $88 \%$ de los niños tuvieron su madre como cuidadora principal. Por último, se recomienda la atención del personal de enfermería con relación a la cantidad y calidad de los registros.

Descriptores: Enfermería pediátrica; Atención de enfermería; Salud del niño; Procedimientos quirúrgicos operativos.

\section{INTRODUÇÃO}

A criança é um ser singular que possui necessidades físicas, psíquicas e emocionais que devem ser reconhecidas e atendidas a fim de que a mesma tenha um crescimento e desenvolvimento adequados. O desenvolvimento de uma criança compreende aspectos referentes a fatores emocionais, sociais e intelectuais, que influenciarão em sua qualidade de vida. A saúde emocional e intelectual da criança deve ser cultivada desde a primeira infância a fim de formar cidadãos felizes e equilibrados. ${ }^{1}$

Sabe-se que a família é a primeira referência para a criança. É nela que ela se desenvolve, cresce, adquire hábitos e socializa-se. O grupo familiar é constituído por pessoas com individualidades distintas. Assim, a família deve ser avaliada em seu mundo interior, considerando as diferenças culturais, valores e crenças bem como as redes que possibilitam o suporte social e o compromisso perante a sociedade e o poder público. ${ }^{2}$

Tratando-se de crianças, a família tem papel fundamental, tanto para os cuidados quanto para a continuidade e adesão ao tratamento. o cuidado centrado na criança e sua família surgiu devido à compreensão de que a família é um elemento fundamental durante a reabilitação e no processo de cuidar evitando o isolamento social que pode contribuir como fator de risco, em especial na infância. ${ }^{3}$

A experiência da hospitalização revela-se para a criança, desde a mais tenra idade, como uma situação traumática pela representação que assumem os significados de dor, sofrimento e dificuldades, na maioria das vezes incompreensíveis ao viver do infante. ${ }^{4}$

A hospitalização suscita uma mobilização dos familiares, tornando-se necessário a reorganização familiar para vivenciar todas as fases deste processo. Nesse sentido, busca-se transmitir um sentimento de confiança à criança, posto que ela reage conforme o contexto em que está inserida e a forma como se interrelaciona.

Em algumas situações de internação pediátrica pode haver a necessidade da realização de algum procedimento cirúrgico. Além dos efeitos negativos comuns à hospitalização, ao se submeter a uma cirurgia a criança está exposta ao estresse que o processo cirúrgico desencadeia. Assim, a assistência de enfermagem à criança internada 


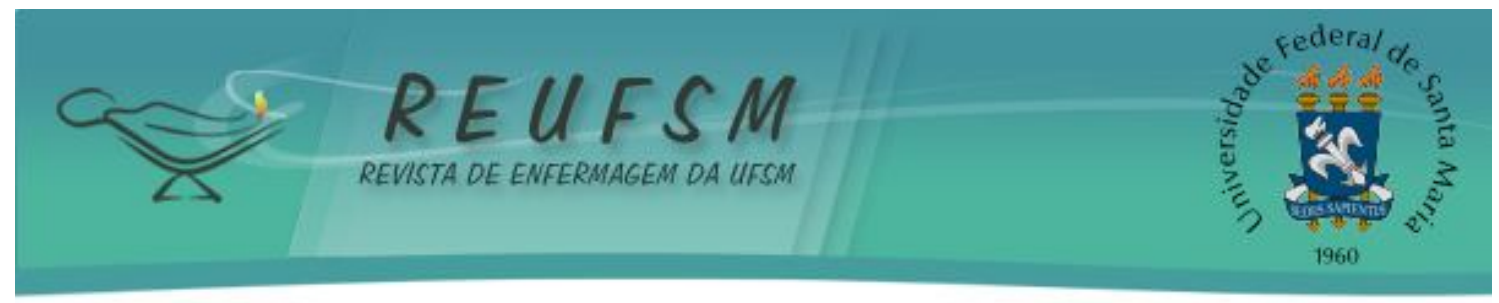

para tal procedimento deve ter como objetivos básicos minimizar o estresse pré-operatório e da própria hospitalização e assegurar uma rápida e efetiva recuperação pós-operatória. ${ }^{5}$

A criança internada para realização de cirurgia pode sentir sua corporeidade ameaçada. Nesse sentido cabe ao enfermeiro ampliar a assistência e o cuidado para além da técnica cirúrgica, considerando que durante a realização dos procedimentos a criança precisa reconstruir-se para enfrentar a nova realidade posta. ${ }^{6}$

Além disso, a realização de uma cirurgia pode gerar uma crise na criança e para sua família, tendo em vista as representações populares e os mitos que existem acerca dos procedimentos cirúrgicos devido às experiências anteriormente conhecidas sobre 0 procedimento. Esta afirmação é confirmada em um estudo realizado em 2007 em que a atenção a família é enfatizada, salientando que o compromisso de cuidar de uma criança pode gerar crises familiares. ${ }^{7}$

Assim, o cuidar em pediatria precisa englobar a inclusão da família, buscando a participação conjunta, pois a presença do familiar é fonte de segurança e afeto e beneficiam a criança, possibilitando a adaptação do infante no âmbito hospitalar. Além dos procedimentos e técnicas, o apoio e as orientações à criança e a sua família precisam estar presente no planejamento dos cuidados.

A enfermagem deve organizar a prática assistencial a partir de situações singulares, respeitando as limitações da família e propiciando autonomia desta em relação ao cuidado prestado a criança, em especial, após a alta hospitalar ${ }^{8}$.

Os profissionais da saúde, principalmente, a equipe de enfermagem, por ter maior proximidade durante o cuidado à criança, precisa reconhecer e valorizar o binômio criança/família. Desta forma, o papel da equipe de enfermagem deve não somente se deter à realização dos procedimentos técnicos, mas também assistir a família em suas dúvidas, apoiar as suas iniciativas e oferecer constante estímulo no desenvolvimento dos cuidados relacionados à criança, sempre tendo em vista o contexto físico, socioeconômico, culturais e espirituais da família. ${ }^{9}$

Neste contexto, cuidar da criança e de sua família significa compreendê-la em seu processo de viver e estar atento ao seu modo de enfrentamento diante da doença, da hospitalização e principalmente do estresse perioperatório que permeia todos os envolvidos. Assim a assistência de enfermagem à criança cirurgiada deve ter como objetivos básicos minimizar o estresse pré-operatório, a própria hospitalização e assegurar uma rápida e efetiva recuperação pós-operatória. ${ }^{10}$

Este estudo justifica-se pela necessidade de conhecer os aspectos sócio-clínico das crianças que internaram para tratamento cirúrgico a fim de organizar/planejar a assistência de enfermagem tendo em vista o cuidado centrado na família. Deste modo, este estudo teve por objetivo caracterizar crianças submetidas a tratamento cirúrgico em um hospital de ensino no sul do Brasil.

\section{MÉTODO}

Trata-se de uma análise documental quantitativa de caráter descritivo e retrospectivo. Os cenários do estudo incluíram os serviços pediátricos de internação: Pronto Socorro Pediátrico (PSP), Unidade de Internação Pediátrica (UIP), Centro de Tratamento da Criança com Câncer (CTCRIAC) e a Unidade de Terapia Intensiva Pediátrica (UTIP) do Hospital Universitário de Santa Maria (HUSM). A população desse estudo foram as crianças entre 28 dias a 12 anos de idade internadas nas unidades supracitadas durante 0 ano de 2006 para realização de cirurgia, totalizando 272 crianças. Do total de prontuários calculou-se uma amostra estatística de 107 prontuários, considerando uma margem de erro de 5\% 


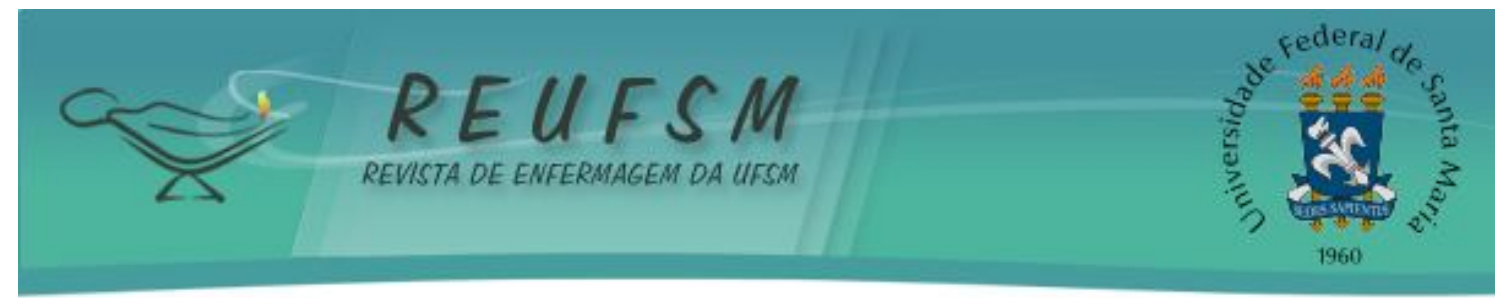

Os prontuários disponíveis no Serviço de Arquivo Médico (SAME) da referida instituição hospitalar foram selecionados de maneira aleatória para a coleta de dados. Sendo incluídos prontuários de crianças que estiveram internadas na instituição para tratamento cirúrgico entre 01 de janeiro a 31 de dezembro de 2006. Em caso de haver mais de uma intervenção cirúrgica no período foram analisadas as informações contidas nos prontuários referentes à última internação.

Para a coleta dos dados que ocorreu nos meses de setembro e outubro de 2008 foi utilizado um formulário próprio, testado previamente, contendo as seguintes variáveis: dados de identificação da criança (iniciais do nome, data de nascimento, sexo, registro da instituição, procedência); dados da internação (tipo de procedimento cirúrgico, história diagnóstica pregressa, história diagnóstica atual, número de internações, tempo de internação, patologias associadas, intercorrências anteriores, local de destino pós alta). Os dados foram submetidos a tratamento estatístico, utilizando-se distribuição de frequências absolutas e relativas.

O projeto de pesquisa foi aprovado pelo Comitê de Ética em Pesquisa da instituição (CEP/ UFSM) sob número de protocolo: 0130. 0.243.000-08.

\section{RESULTADOS E DISCUSSÃO}

Quanto ao sexo, o número de meninos que internaram para realização de cirurgias foi de 60 (56\%) e o número de meninas de 47 (44\%). Pode-se relacionar esse dado ao fato de que a apendicite foi o agravo recorrente nesse estudo, sendo que esta acomete mais 0 sexo masculino, ocorrendo, principalmente em crianças em idade escolar e pré-escolar e de procedência rural. ${ }^{11}$

Em relação à faixa etária das crianças internadas no período, foram categorizadas da seguinte forma: lactentes (28 dias a dois anos), pré-escolares (três a cinco anos) e escolares (seis a 12 anos) conforme Gráfico 1 a seguir:

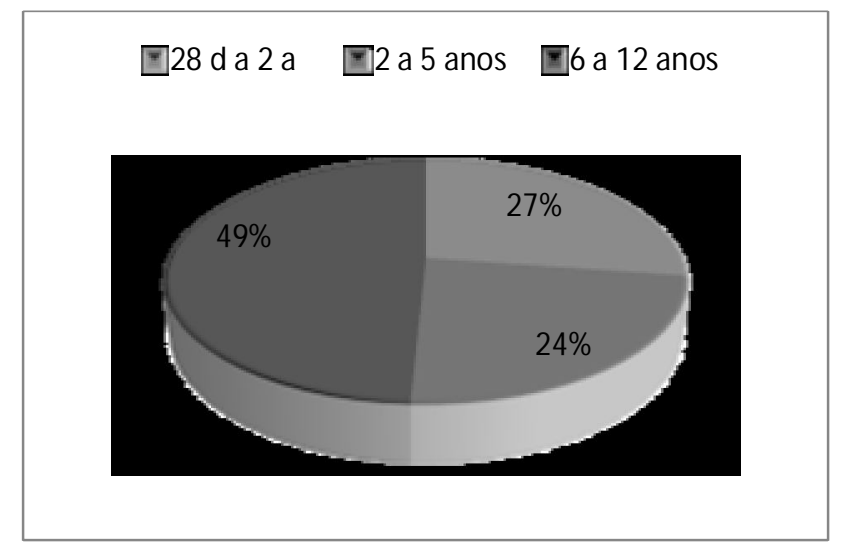

Gráfico 1- Distribuição da faixa etária das crianças internadas para tratamento cirúrgico. Santa Maria, 2006.

Observa-se que a metade das crianças 52 (49\%) que internaram para realização de cirurgias no período proposto, estava em idade escolar. Já as crianças em idade préescolar, de três a cinco anos, totalizaram $24 \%$ assim $73 \%$ das crianças encontrava-se em idade pré-escolar e escolar.

As crianças em idade escolar predominaram na amostra, corroborando com um estudo desenvolvido em 2007 que apontou como principais causas de hospitalização de crianças de cinco a nove anos a apendicite, as pneumonias e os traumatismos. ${ }^{12}$ 


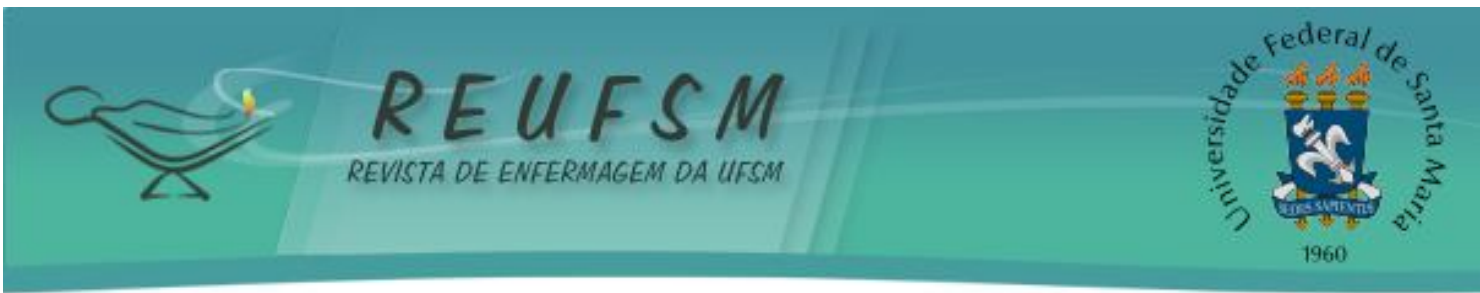

Para apresentar a procedência das crianças, dividiu-se o estado do Rio Grande do Sul em regiões geográficas.

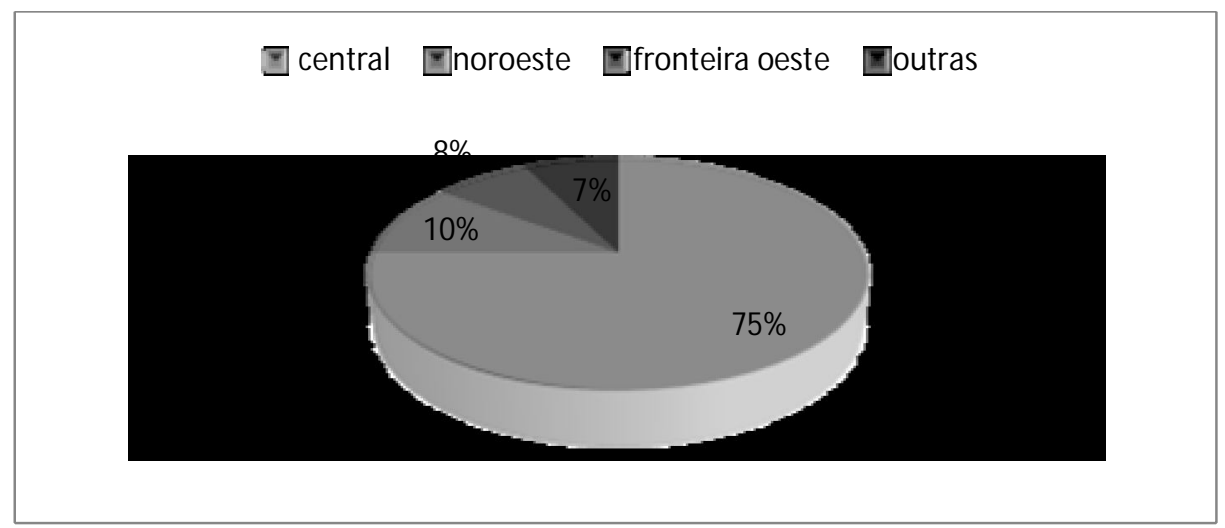

Gráfico 2- Procedência das crianças internadas para tratamento cirúrgico. Santa Maria, 2006.

Assim, 75\% delas procederam da região central. Isto se deve, em parte, ao fato de que a instituição é referência na área da cirurgia pediátrica.

Já para a variável história diagnóstica, conforme último registro no prontuário das crianças pesquisadas destaca-se que algumas delas apresentaram mais de uma patologia como diagnóstico, totalizando 124 diagnósticos registrados, conforme Tabela 1 a seguir:

Tabela 1: História Diagnóstica conforme registro no último prontuário das crianças internadas para tratamento cirúrgico. Santa Maria, 2006. N=124

\begin{tabular}{l|cc}
\hline Diagnóstico & $\mathrm{N}$ & $\%$ \\
\hline Infecções do trato respiratório & 22 & $18 \%$ \\
Derrame pleural & 10 & $8 \%$ \\
Apendicite & 35 & $28 \%$ \\
Infecções do trato urinário & 3 & $2,4 \%$ \\
Tumores & 19 & $15 \%$ \\
Patologias do sistema digestivo & 7 & $6 \%$ \\
Patologias congênitas & 16 & $13 \%$ \\
Patologias infecciosas & 5 & $4 \%$ \\
Traumas & 4 & $3,2 \%$ \\
Outras & 3 & $2,4 \%$ \\
\hline
\end{tabular}

Nesse panorama, a apendicite aguda prevaleceu com $28 \%$ seguida das infecções do trato respiratório com $18 \%$ Já os tumores e as patologias congênitas apresentaram, respectivamente, $15 \%$ e $13 \%$

Apendicite aguda é a doença que mais comumente requer cirurgia abdominal de emergência na criança, constituindo a principal causa de abdômen agudo cirúrgico na criança maior de dois anos de idade e é responsável por cerca de $10 \%$ de todas as admissões em salas de emergências pediátricas. ${ }^{13}$

As infecções do trato respiratório, agudas ou crônicas, são consideradas importantes causas de morbimortalidade infantil, sendo as infecções respiratórias agudas 0 maior motivo de procura nos serviços de saúde e internações de crianças, como a pneumonia. ${ }^{14}$

Quanto aos procedimentos cirúrgicos realizados nas 107 crianças que tiveram os prontuários analisados, obteve-se que a apendicectomia foi realizada em 33\% dos casos, 


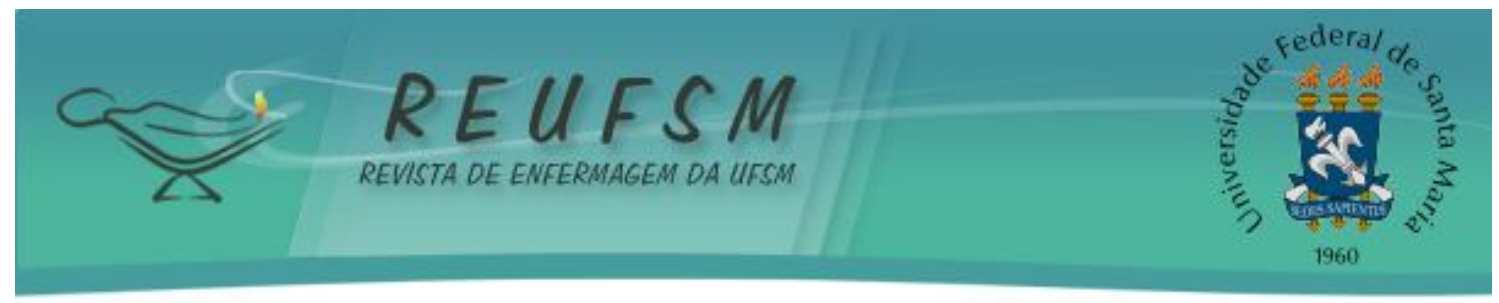

seguida da ressecção de tumores com $18 \%$ e a drenagem de tórax com $11 \%$ Vale ressaltar que a drenagem de tórax foi realizada, principalmente, para 0 tratamento de complicações referentes às infecções do trato respiratório.

O derrame pleural é um achado frequente nos casos de infecções do trato respiratório, como a pneumonia. Essa complicação é causada em geral por uma pneumonia e algumas crianças necessitam de drenagem cirúrgica para tratá-la. ${ }^{15}$

A variável tempo de internação das crianças em tratamento cirúrgico revela que $37 \%$ das crianças que realizaram cirurgias permaneceram internadas até cinco dias, já $25 \%$ das crianças ficaram internadas de 6 a 10 dias, 22\% estiveram internadas de 11 a 15 dias e $17 \%$ internaram por mais de 15 dias. 0 tempo de permanência das internações, principalmente das cirúrgicas, vem diminuindo nos últimos anos em função das inovações tecnológicas que vêm ocorrendo na assistência à saúde. ${ }^{16}$

As condições clínicas e o diagnóstico foram fatores relevantes para que 71 das 107 crianças analisadas internassem em outras unidades do hospital além da unidade de internação pediátrica (UIP), como a unidade de terapia intensiva pediátrica (UTIP) e 0 pronto socorro pediátrico (PSPed).

Quanto às demandas de cuidados na alta hospitalar das crianças cirúrgicas, a retirada de pontos destacou-se, seguida dos cuidados com dispositivos invasivos. Ressaltase que não foram encontrados registros do envolvimento da equipe de enfermagem com a alta dessas crianças com necessidades de demandas de cuidados. No momento da alta hospitalar o enfermeiro deve estar atento as condições sociais, emocionais e dos recursos disponíveis à criança e também avaliar o conhecimento do cuidador/familiar quanto aos cuidados no domicílio. Nesse sentido, considera-se imprescindível o envolvimento do enfermeiro no processo de alta da criança com vistas à continuidade de assistência no domicílio. ${ }^{17}$

Atualmente, verifica-se um crescimento no número crianças portadoras de alguma necessidade especial de saúde ou doença crônica que fazem uso de algum tipo de equipamento ou tecnologia avançada para sua sobrevivência. Entretanto, nesse estudo 95\% das crianças internadas para realização de cirurgias no ano de 2006 não apresentaram necessidades especiais de saúde. Pode-se associar o baixo percentual (5\%) com o fato de que as CRIANES (crianças com necessidades especiais) não internam para tratamento cirúrgico. Sabe-se que a principal causa de internação de CRIANES no município cenário da pesquisa são as infecções respiratórias agudas, portanto elas representam um caso clínico e não cirúrgico. ${ }^{18}$

Os achados da pesquisa referentes às necessidades especiais de saúde tiveram sua classificação quanto às demandas de cuidados em: cuidados tecnológicos, cuidados medicamentosos, demandas de desenvolvimento e cuidados habituais modificados. ${ }^{19}$ Assim, foram identificados dois desses grupos: os dependentes de tecnologia: uso de cateter semiimplantado, cateter totalmente implantado, gastrostomia, sondagens vesicais de alívio e bolsa de colostomia; e demanda de cuidados medicamentosos, as criança com HIV/ AIDS.

Nessa pesquisa não foi encontrado registros de orientações prestadas aos familiares ou acompanhantes das crianças com necessidades especiais de saúde para que houvesse continuidade ao tratamento e aos cuidados no domicílio. Entretanto, destaca-se que cabe ao enfermeiro a avaliação das necessidades da criança e da família. Tal avaliação não deve ser feita de forma simplista, direcionada apenas para necessidades físicas e individuais. É necessário que se faça uma avaliação mais ampla dessas necessidades, considerando-se aspectos emocionais, financeiros e 0 ambiente familiar. ${ }^{17}$

Para que a enfermagem desenvolva um cuidado integral em pediatria, faz-se necessário valorizar, interagir e conhecer os familiares/cuidadores das crianças 


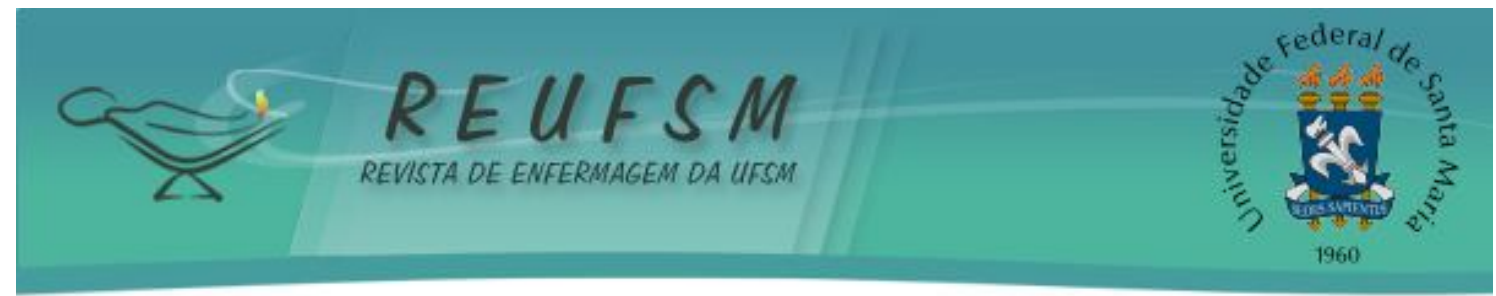

hospitalizadas, por meio do diálogo entre familiar e equipe, a fim de aperfeiçoar o plano de cuidados, para a criança, levando em consideração a realidade da dinâmica familiar. ${ }^{20}$

Em relação ao cuidador principal das crianças, a mãe destacou-se, sendo que $88 \%$ estavam acompanhadas pela mãe, $10 \%$ pelo pai e $2 \%$ pela avó. Esse dado confirma o que outros estudos tem apresentado sobre a idealização da mulher como a figura da boa mãe. ${ }^{21}$ O papel materno idealizado foi socialmente construído como padrão de comportamento das mulheres, onde a necessidade de maternagem era uma característica universal feminina, fazendo parecer como um dom, um sentimento instintivo e estritamente biológico das mulheres, independente da cultura ou das condições sócio-econômicas. ${ }^{21}$ Aquelas que não possuíam o desejo de maternar fugiam à norma, sendo classificadas como exceções patológicas ou desvios. ${ }^{22}$

Uma das limitações do estudo foi à falta de informações nos prontuários, pois os registros de enfermagem enfatizavam os cuidados rotineiros com anotações repetitivas e ausência de informações referentes às orientações prestadas aos familiares, por exemplo. Isto denota certa invisibilidade das ações de enfermagem que, por vezes, não registrando as ações executadas, desvaloriza e fragiliza o papel do enfermeiro na prática.

\section{CONCLUSÕES}

Dentre as crianças que internaram para realização de cirurgias em 2006, tiveram maior frequência àquelas do sexo masculino, na faixa etária de seis a 12 anos, procedentes da região central do estado do Rio Grande do Sul. Os diagnósticos médicos prevalentes estiveram relacionados à apendicite e as infecções do trato respiratório e, assim, os procedimentos cirúrgicos de maior destaque foram apendicectomia e toracotomia para drenagem de tórax.

A maior parte das cirurgias foi simples e as crianças ficaram internadas em torno de cinco dias, principalmente para o tratamento com antibióticos. As mães destacaram-se como cuidadoras principais.

Constatou-se que foi maior o número de crianças internadas na UIP em contraponto com a UTIP. Tal fato pode indicar que foram poucas as cirurgias de grande porte e poucos casos agravaram-se. No PS PED foram atendidas $57 \%$ das crianças. Também 0 fato de algumas cirurgias terem sido previamente agendadas contribuiu para que as crianças internassem diretamente na UIP.

Em relação às demandas de cuidados na alta hospitalar e a presença de possíveis necessidades especiais de saúde, essas não foram significantes na população estudada.

Este estudo possibilitou ao serviço de saúde conhecer a clientela atendida, a fim de contribuir para a organização e o planejamento da assistência prestada às crianças em tratamento cirúrgico. Também, suscitou reflexões sobre a importância do envolvimento da família no processo de cuidar da criança em tratamento cirúrgico, apontando para uma prática de enfermagem centrada na família.

Por fim, recomenda-se atenção da equipe de enfermagem em relação à quantidade e qualidade dos registros nos prontuários.

\section{REFERÊNCIAS}

1 Gallo DL, Trella CS. Humanizando a Pediatria: Relato de Experiência do Trabalho Voluntário. Londrina : Universidade Estadual de Londrina ; 2007. Disponível em: http:/ / www. ccs. uel. br/ espacoparasaude/ v4n1/ doc/ pediatria. doc. html. 


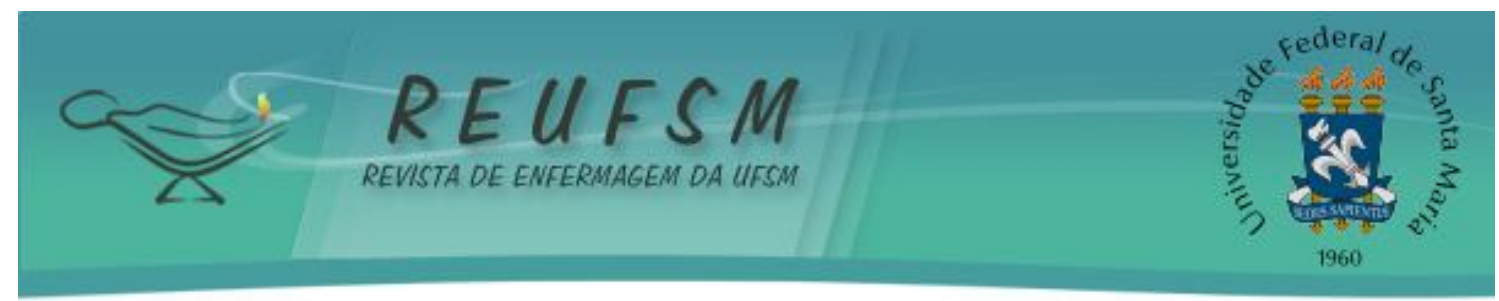

2 Próspero ENS, Machado H, Stuker H, Elsen I, Ângelo MV, Silva YF. Riscos potenciais de saúde: análise de exclusão e inclusão social das famílias de Itajaí/SC. Texto e Contexto Enferm. 2005; 14(Esp): 47-53.

3 Pinto J P, Ribeiro CA, Pettengill MM, Balieiro MMFG. Cuidado centrado na família e sua aplicação na enfermagem. Rev Bras Enferm. 2010; 63(1): 132-135.

4 Issi BH, Jacoby AMR, Lima EC, Wilsmann J, Wulle JD, Cachafeiro MEH et al. Em foco a família: a construção de uma trajetória da enfermagem pediátrica do Hospital de Clínicas de Porto Alegre. Rev HCPA. 2007; 27(2): 39-42.

5 Favero L, Dyniewicz AM, Spiller APM, Fernandes LA. A promoção do brincar no contexto da hospitalização infantil como ação de enfermagem: relato de experiência. Rev Cogitare Enferm. 2007; 12(4): 519-524.

6 Motta MGC. O entrelaçar de mundos: Família e hospital: O viver em família e a sua interface com a saúde e a doença. 2ª ed. Maringá: Ed. UEM; 2004.

7 Leite NSL, Cunha SR. A família da criança dependente de tecnologia: aspectos fundamentais para a prática de enfermagem no ambiente hospitalar. Esc Anna Nery Rev Enferm. 2007; 11(1): 92-97.

8 Pimenta EAG, Collet N. Dimensão cuidadora da Enfermagem e da família na assistência à criança hospitalizada: concepções da Enfermagem. Rev Esc Enferm. 2009; 43(3): 622-629.

9 Souza TV, Oliveira ICS. Interação familiar/ acompanhante e equipe de enfermagem no cuidado à criança hospitalizada: perspectivas para a Enfermagem Pediátrica. Esc Anna Nery Rev Enferm. 2010; 14(3): 551-559.

10 Favero L, Dyniewicz AM, Spiller APM, Fernandes LA. A Promoção do brincar no contexto da hospitalização infantil como ação de enfermagem: Relato de experiência. Rev Cogitare Enferm. 2007; 12(4): 519-524.

11 Reis J M, Oliveira DCN, Lucatto TM, Júnior WBR. Diagnóstico e tratamento de 300 casos de apendicite aguda em crianças e adolescentes atendidos em um hospital universitário. Rev Médica de Minas Gerais. 2008; 18(1): 11-15.

12 Vargas AN, Quezada A. Epidemiología, nueva morbilidad pediátrica y rol del pediatra. Rev Chilena Pediatría. 2007; 78(Supl. 1): 103-110.

13 Vital FP, Martins LJ. Estado atual do diagnóstico e tratamento da apendicite aguda na criança: avaliação de 300 casos. Rev Col Bras. 2005; 32 (6): 310-315.

14 Rosa AM, Ignotti E, Hacon SS, Castro HA. Análise das internações por doenças respiratórias em Tangará da Serra - Amazônia Brasileira. J Bras Pneumol. 2008;34(8):575582.

15 Freitas S, Fraga JC, Canani F. Toracoscopia em crianças com derrame pleural parapneumônico complicado na fase fibrinopurulenta: estudo multi-institucional. J Bras Pneumol. 2009; 35(7): 660-668.

16 Carvalho BG. Internações hospitalares em Londrina: 1990-1998 [dissertação de mestrado em Saúde Coletiva]. Londrina: Centro de Ciências da Saúde, Universidade Estadual de Londrina; 2000.

17 Madeira LM. Alta hospitalar da criança: implicações para enfermagem. São Paulo : Faculdade de Saúde Pública: Universidade de São Paulo; 2008. 


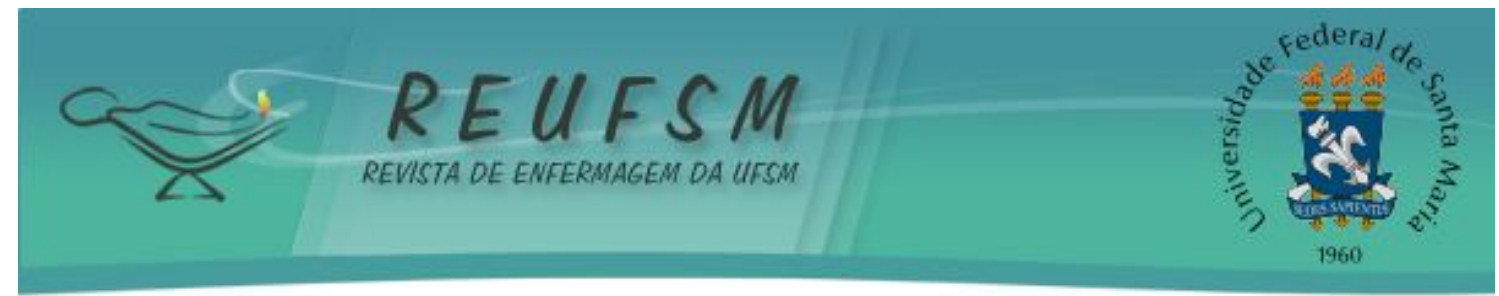

18 Vernier ET N, Cabral IE. Caracterização de crianças com necessidades especiais de saúde e seus familiares cuidadores, Santa Maria (RS), 2004-2005: subsídios para intervenções de enfermagem Rev Soc Bras Enferm Ped. 2006; 6(1): 37-45.

19 Cabral IE, Moraes JRMM, Santos FF. O egresso da terapia intensiva neonatal de três instituições públicas e a demanda de cuidados especiais. Esc Anna Nery Rev Enferm. 2003; 7(2): 211-218.

20 Góes FGB, La Cava AM. Práticas educativas em saúde do enfermeiro com a família da criança hospitalizada. Rev Eletr Enf. [Internet]. 2009; 1(4): 942-945.

21 Neves ET, Cabral IE. Empoderamento da mulher cuidadora de Crianças com Necessidades Especiais de Saúde. Texto Contexto Enferm. 2008; 17(3): 552-560.

22 Tourinho J. A mãe perfeita: idealização e realidade. Rev IGT na Rede. 2006; 3(5): 1-33.

Data de recebimento: 20/01/2011

Data de aceite: 06/04/2011

Contato com autora responsável: Andressa da Silveira.

Endereço: Rua J oão Atílio Zampieri, 132/ 301, Bairro Camobi. Santa Maria, RS.

E-mail: andressadasilveira@gmail.com 